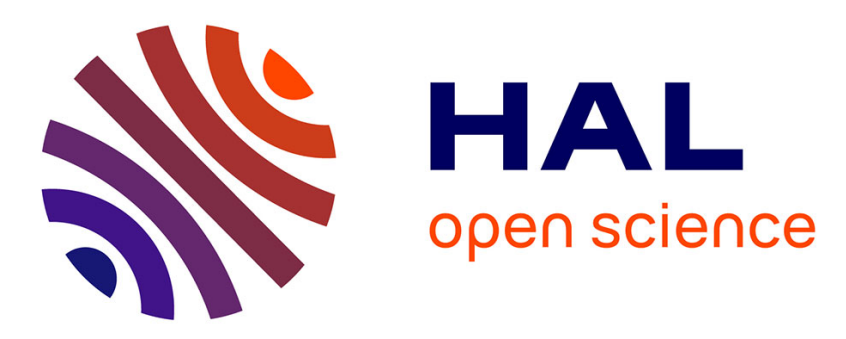

\title{
Estimating Center of Mass Trajectory in Quiet Standing: a Review
}

\author{
Alice Nicolaï, Julien Audiffren
}

\section{To cite this version:}

Alice Nicolaï, Julien Audiffren. Estimating Center of Mass Trajectory in Quiet Standing: a Review. 2019 41st Annual International Conference of the IEEE Engineering in Medicine \& Biology Society (EMBC), Jul 2019, Berlin, Germany. pp.6854-6859, 10.1109/EMBC.2019.8857888 • hal-03140399

\author{
HAL Id: hal-03140399 \\ https://hal.science/hal-03140399
}

Submitted on 15 Mar 2021

HAL is a multi-disciplinary open access archive for the deposit and dissemination of scientific research documents, whether they are published or not. The documents may come from teaching and research institutions in France or abroad, or from public or private research centers.
L'archive ouverte pluridisciplinaire HAL, est destinée au dépôt et à la diffusion de documents scientifiques de niveau recherche, publiés ou non, émanant des établissements d'enseignement et de recherche français ou étrangers, des laboratoires publics ou privés. 


\title{
Estimating Center of Mass Trajectory in Quiet Standing: a Review
}

\author{
Alice Nicolaï ${ }^{1,2}$ and Julien Audiffren ${ }^{1,2}$
}

\begin{abstract}
As falls prevalence increases with the aging of the population, early detection of balance degradation is of great importance for efficient prevention and treatment. This work compares a wide range of state-of-the-art methods to estimate the trajectory of the center of mass - a key aspect of postural control quantification. This comparison is done through multiple complementary metrics over a large dataset, highlighting the pros and cons of each method as well as the key influence of the data preprocessing. Additionally, we introduce several improvements and parameter tunings for these methods that increase their accuracy or reduce contextual information requirement.
\end{abstract}

\section{INTRODUCTION}

Falls of older adults are considered a major public health problem [1] due to their severe consequences [2] and their prevalence - each year more than a third of population 65 years-old and older faces a fall [3]. Therefore, early detection of balance deterioration is key, as prevention strategies have shown encouraging results [4]. Since these strategies have been shown to be more effective when targeted at vulnerable individuals, accurate quantification of balance control has attracted significant attention in the recent years.

Quantifying Balance: Balance is achieved through the complex synergy of many biological systems, including the visual, proprioceptive, vestibular and central nervous systems [5] - and is therefore difficult to thoroughly quantify. Postural control - defined as the capacity to stand still without falling - is considered an important component of balance [6] and can be tested to evaluate the risk of falling, through observational tests or measurements (see e.g. [7]). Postural control is generally measured by recording the displacement of the center of pressure $(\mathrm{CoP})$ - the point of application of the ground reaction forces resultant under the feet [8] - using a force platform [9]. The resulting signal, usually called a statokinesigram, describes the movements of the $\mathrm{CoP}$ which are assumed to be the consequences of the neuromuscular response to control the position of the center of mass (CoM) [10]. Following this idea, previous works have shown that the dynamics of both the CoM and the CoP are crucial to the quantification of postural control [11], [10], [8].

However, while the $\mathrm{CoP}$ may be recorded using even the simplest force platform [12], the CoM location cannot be directly measured [13]. Therefore, there has been an increased interest in the recent past on methods that allow to estimate the CoM position using only force platforms.

\footnotetext{
${ }^{1}$ Cognition and Action Group, Cognac-G, CNRS UMR 8257, Université Paris Descartes, Sorbonne Paris Cité, Service de Santé des Armées, 45 rue des Saints Pères, 75006 PARIS, France. ${ }^{2}$ Centre de mathématiques et de leurs applications, CNRS, ENS ParisSaclay, Université Paris-Saclay, 94235, Cachan cedex, France. Email: alice.nicolaidcmla.ens-cachan.fr
}

Related Work: The problem of estimating the position of the CoM during quiet standing is hardly new, and many approximations have been proposed, the most frequently used of which are described below.

The pioneering approach consists in the measurement of the 3D-movements of the body with the tracking of markers placed on different parts of the body [14]. The latter method, referred to as the kinematic method or segmental analysis, is often considered the gold-standard method [15], [16], [17], [18].

However, this approach suffers from several drawbacks, including the complexity of the kinematic protocol and the cost of the sensors. Numerous following studies have proposed alternative methods to estimate the position of the $\mathrm{CoM}$ directly from the force platforms signals. Two main families of CoM approximation strategies can be found in the literature. Methods of the first family use the double integration of the horizontal ground reaction force to derive the CoM positions from the CoP trajectory [19], [20]. The second family does not require the recording of horizontal ground reaction forces - which is an interesting advantage considering the increasing popularity of low cost force platforms such as the Wii Balance Board [12] - and instead embeds the relation between the $\mathrm{CoP}$ and the $\mathrm{CoM}$ in the frequency domain to estimate the CoM trajectory [21], [16].

Multiple previous works have compared several of these methods in order to find the best one [8], [13], [16]. Yet, those studies generally compare a few of the methods in the aforementioned families, use varying preprocessing treatments of the signals and evaluate the different estimations on small populations. Moreover, we think that several of the parameters could be improved through an optimization process over a large dataset.

Contributions: In this work we compare a wide range of state-of-the-art methods designed to approximate the position of the CoM. We compare all of them to the kinematic method, which is considered a gold standard for this task [18]. To evaluate each method, we use an extensive collection of metrics on a large data set with kinematic and ground reaction forces of human balance [22] - which contains more than 500 recordings in multiple conditions. We highlight the pros and cons of each method and in particular we shed light on the influence of the pretreatment of the signals, which is key for forces-based approaches. Finally, we introduce several improvements and parameter tunings for some of the commonly used algorithms that either significantly increase the approximation performance or reduce the quantity of contextual information required (e.g. biodata). 


\section{Model And Algorithms}

In this section we first describe the state of the art approaches to estimate the CoM position and discuss the possible improvements to these methods.

Notation: We use $m(t)$ (resp. $x(t), \hat{m}(t)$ ) to denote the position of the CoM (resp. CoP, CoM approximation) in the horizontal plane at time $t$. Additionally, $\tilde{m}_{f}$ (resp. $\tilde{x}_{f}$ denotes the coefficient associated with frequency $f$ in the Discrete Fourier Transform (DFT) of $m$ (resp. $x$ ). Finally, $F_{h}(t)$ represents the projection of the ground reaction force at time $t$ in the horizontal plane and $F_{v}(t)$ denotes its vertical component.

\section{A. Kinematic Method (KM)}

This approach uses markers that track the position of different parts of an individual body. Each part $i$ is considered a rigid segment in order to deduce its own CoM $m^{i}(t)$. Then, the global CoM position $m(t)$ is computed as the weighted average of the CoM of each body part

$$
m(t)=\sum_{i} c_{i} m^{i}(t)
$$

where the $c_{i}$ are chosen according to [23].

This is often considered as the method of reference [15], [16], [17], [18] since the sensors involved in the markers tracking generally have great accuracy $(\leq 0.1 \mathrm{~mm})$. Therefore, and following previous works [16], we will compare the other methods to the kinematic technique to evaluate their performances.

\section{B. Force Integration (FI)}

These methods derive the position of the CoM from the double integration of its acceleration - deduced from the horizontal ground reaction force using Newton's second law. Formally,

$$
\hat{m}(t)=m_{0}+\int_{s=0}^{t}\left(\dot{m}_{0}+\int_{r=0}^{s} \frac{F_{h}(r)}{M} d r\right) d s
$$

where $\mathrm{M}$ is the weight of the subject. Therefore it requires the knowledge of the initial position $m_{0}$ and speed $\dot{m}_{0}$ of the CoM. Two main techniques are used to compute those initial conditions [13].

1) Global Second Integral (Global SI): This approach [19] estimates the initial constants of (1) by using a rough approximation of the $\operatorname{CoM} E(t)$, which is defined as:

$$
E(t)=x(t)+\frac{h(t)}{F_{z}(t)} F_{h}(t)
$$

where $h(t)$ is the height of the CoM at time $t . m_{0}, \dot{m}_{0}$ are chosen to minimize

$$
\int_{t}\|\hat{m}(t)-E(t)\|_{2}^{2} d t
$$

by using a least square regression.
2) Zero-point-to-Zero-point Double Integration (ZZDI): One of the main difficulty of Global SI is that (2) requires the knowledge of $h(t)$. To circumvent this problem, an other method to compute the initial conditions is proposed in [20]. This approach uses the assumption that the CoM and the $\mathrm{CoP}$ are at the same location on one horizontal axis if and only if the component of the ground reaction force on this axis equals zero. Then, the CoM position between two such successive intersection points $t_{i}, t_{i+1}$ is computed as the double time integration of the horizontal forces, where the constants of integrations are chosen such that $x$ and $\hat{m}$ coincide in both $t_{i}$ and $t_{i+1}$. It should be noted that in this method the initial conditions change in each interval $\left[t_{i}, t_{i+1}\right]$, where (1) becomes:

$$
\hat{m}(t)=x\left(t_{i}\right)+\int_{s=t_{i}}^{t}\left(\dot{m}_{t_{i}}+\int_{r=t_{i}}^{s} \frac{F_{h}(r)}{M} d r\right) d s
$$

with

$$
\dot{m}_{t_{i}}=\frac{1}{t_{i+1}-t_{i}}\left(x\left(t_{i+1}\right)-x\left(t_{i}\right)-\int_{s=t_{i}}^{t_{i+1}} \int_{r=t_{i}}^{s} \frac{F_{h}(r)}{M} d r d s\right)
$$

As $F_{h}$ is rarely exactly zero, we determined zero-crossings by searching for points where the horizontal force curve switches from negative to positive values as proposed in [20].

\section{Frequency-Based Estimation (FBE)}

This family of methods relies on the relation between the $\mathrm{CoP}$ and the CoM in the frequency domain. Indeed, the CoM average position is supposed to be close to the CoP average position and only high frequency components of the two trajectories are assumed to differ significantly [24], [16]. Following this idea, the CoM DFT is derived from the CoP DFT by using different variations of the low pass filter principle, which states that the ratio of $\tilde{m}_{f}$ to $\tilde{x}_{f}$ should be a decreasing function of $f$.

1) Traditional low-pass filters: Two classical version of the low-pass filter have been considered in the literature: $\hat{m}_{t}$ is obtained from $x_{t}$ using either a Cutoff or a second order butterworth low-pass filter (LPF). Commonly used thresholds are $0.4,0.5$ and $0.75 \mathrm{~Hz}$ [16], [18].

2) Low-pass filter derived from anthropometric data (LPFA): A tuned version of the LPF approach has been proposed by [25], [21] that relies on the idea of a natural body frequency [25]. In this model, the ratio between $\tilde{m}_{f}$ and $\tilde{x}_{f}$ is set as

$$
\tilde{m}_{f}=\varphi_{f}^{\mathrm{COP} \rightarrow \mathrm{COM}} \times \tilde{x}_{f}
$$

with

$$
\varphi_{f}^{\mathrm{COP} \rightarrow \mathrm{COM}}=\frac{g h}{g h+\left(h^{2}+C H^{2}\right)(2 \pi f)^{2}},
$$

where $g$ is the gravity constant, $h$ is the average height of the CoM during the recording, $H$ is the height of the individual and $C$ is a constant that depends on the axis $\left(C=5.72 e^{-2}\right.$ for the medio-lateral axis, $C=5.33 e^{-2}$ for the anterioposterior axis) [26]. 

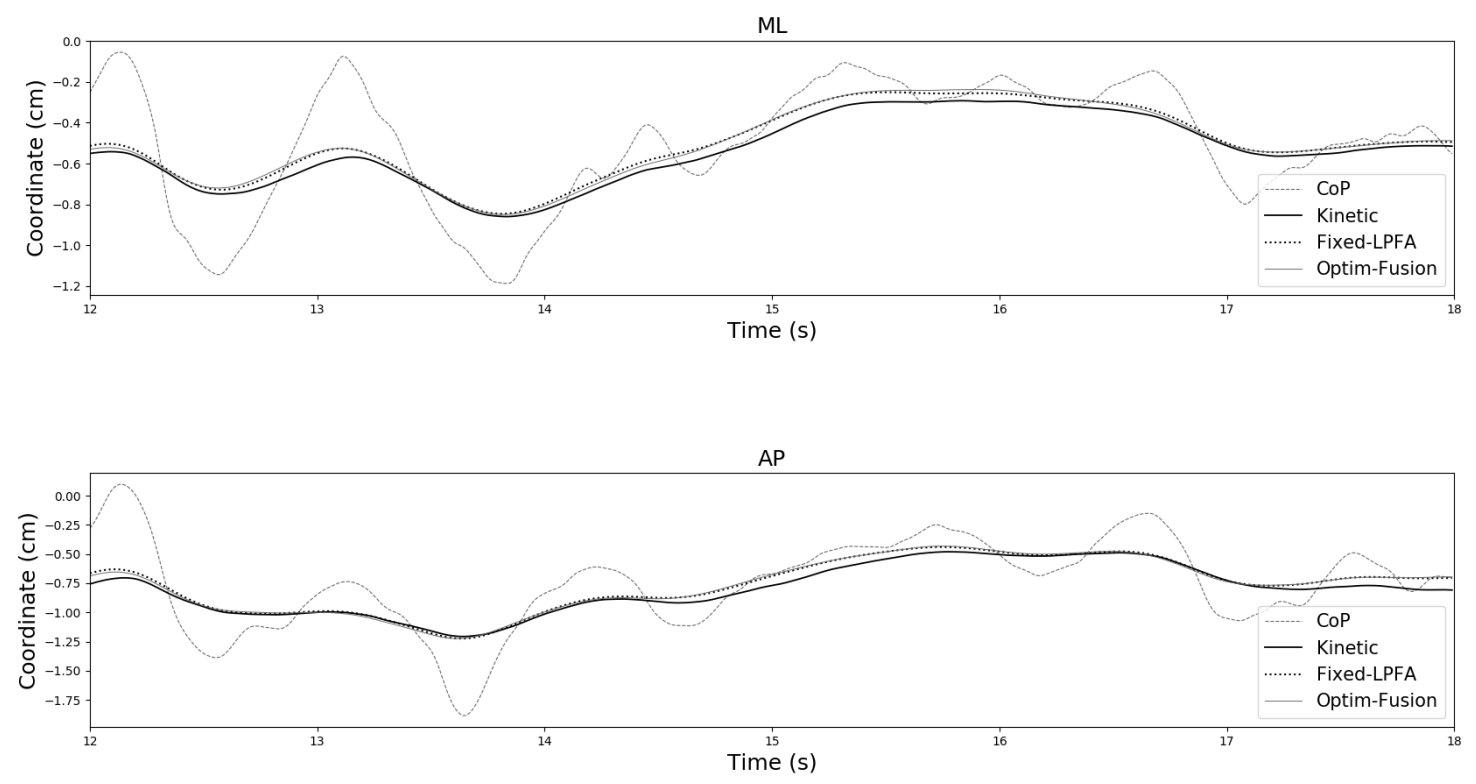

Fig. 1: Example of the trajectories of the CoM estimated by the Kinematic, the Optim-Fusion and the Fixed-LPFA methods. (Up) medio-lateral axis (ML), (down) antero-posterior axis (AP).

Contribution: LPFA with fixed coefficient (Fixed-LPFA): We can rewrite (3) as:

$$
\varphi_{f}^{\mathrm{COP} \rightarrow \mathrm{COM}}=\frac{1}{1+\gamma f^{2}},
$$

where $\gamma$ only depends on several biodata constants of the individual. Moreover, $\gamma$ does not vary greatly $(3.75 \leq \gamma \leq$ 5.1) when computed over all the individuals contained in [22]. Following this remark, we propose a modification of the LPFA method, called Fixed-LPFA, where we set $\gamma=4.2$ - a constant independent of the individual, which appears to produce the best overall performance (see Section III).

3) Moving Average Filter (Boxcar): This method computes the $\mathrm{CoM}$ position as the average of the $\mathrm{CoP}$ points that are close in time [16]. Formally,

$$
\hat{m}(t)=\frac{1}{2 \delta} \int_{t-\delta}^{t+\delta} x(s) d s
$$

where $\delta>0$ is the window radius, a parameter of the model. In their work, [16] have compared the values $0.16,0.25,0.62$ and 0.75 for $\delta$.

\section{Fusion}

The fusion approach, discussed in [27], attempts to overcome the drawbacks of the force integration methods by merging those methods with frequency-based methods, using the idea that the low frequency (resp. high frequency) movements of $m$ should be similar to the low frequency (resp. high frequency) movement of $x$ (resp. $\hat{m}^{\text {ZZDI }}$, the force integration approximation of $m$ presented in Subsection IIB.2). Formally, given a frequency threshold $\tau$, the DFT of $m$ is computed as follows :

$$
\tilde{m}_{f}=\left\{\begin{array}{l}
\tilde{x}_{f} \text { if } f<\tau \\
\tilde{m}_{f}^{\text {ZZI }} \text { otherwise }
\end{array}\right.
$$

In their work, [27] proposed $\tau=0.5 \mathrm{~Hz}$ as the most efficient threshold.

\section{EXPERIMENTAL EVALUATIONS}

\section{A. Dataset}

We start by briefly describing the dataset used in this study, and we refer the reader to [22] for an in-depth presentation. The database contains 49 individuals, including 22 elderly individuals (60 years old and older) and 27 young individuals (38 years old and younger), including a large range of postural control profiles (such as the presence of disability or disease). Each individual was recorded 12 times, standing still for 60 seconds, under different conditions (open eyes, closed eyes, with or without a foam). Each acquisition was recorded using A) two OPT400600-1000 AMTI force platforms to track the $\mathrm{CoP}$ position and the ground reaction forces and B) a Raptor-4 motion analysis system using 12 infrared cameras to track the position of 42 markers and compute the position of the CoM. All the data where filtered with a low-pass Butterworth filter with a $10 \mathrm{~Hz}$ cutoff frequency, fourth order, and zero lag. In order to compare the CoM predicted by the different methods, we used all the $\mathrm{CoP} / \mathrm{CoM}$ pairs of the database indiscriminately, as we are only interested in the CoP/CoM dynamics independently of the individual balance quality. The influence of the individual impairments and acquisition conditions are discussed in Section IV. 
TABLE I: Mean error ( \pm standard deviation) of the Global SI and ZZDI approximations with respect to the kinematic method, using the Pos metric, depending on the high-pass thresholds used for both the CoM and the forces signal.

\begin{tabular}{c|c|c|c} 
High-pass on forces $(\mathrm{Hz})$ & High-pass on CoM $(\mathrm{Hz})$ & ZZDI & Global SI \\
\hline & 0 & $8.13 e^{-02}\left( \pm 1.22 e^{-01}\right)$ & $4.54 e^{-01}\left( \pm 2.45 e^{-01}\right)$ \\
0.1 & 0 & $8.56 e^{-02}\left( \pm 1.23 e^{-01}\right)$ & $5.24 e^{-01}\left( \pm 2.85 e^{-01}\right)$ \\
0.25 & 0.2 & $6.32 e^{-02}\left( \pm 4.69 e^{-02}\right)$ & $3.31 e^{-01}\left( \pm 1.78 e^{-01}\right)$ \\
0.25 & 0.2 & $6.30 e^{-02}\left( \pm 4.72 e^{-02}\right)$ & $1.71 e^{-01}\left( \pm 1.11 e^{-01}\right)$
\end{tabular}

TABLE II: Mean error ( \pm standard deviation) of the approximations of the FI family with respect to the KM, using the different metrics. Optim-Fusion denotes the algorithm with $\tau=0.3 \mathrm{~Hz}$ instead of $0.5 \mathrm{~Hz}$.

\begin{tabular}{|c|c|c|c|}
\hline Metrics & ZZDI & Optim-Fusion & Fusion \\
\hline Pos & $8.56 e^{-02}\left( \pm 1.23 e^{-01}\right)$ & $\mathbf{7 . 5 9 e ^ { - 0 2 }}\left( \pm 1.24 e^{-01}\right)$ & $1.29 e^{-01}\left( \pm 1.37 e^{-01}\right)$ \\
\hline Spd & $3.01 e^{-03}\left( \pm 1.47 e^{-03}\right)$ & 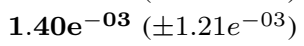 & $4.06 e^{-03}\left( \pm 2.15 e^{-03}\right)$ \\
\hline $\operatorname{VarX}$ & $1.69 \mathbf{e}^{-02}\left( \pm 4.21 e^{-02}\right)$ & $1.83 e^{-02}\left( \pm 4.53 e^{-02}\right)$ & $5.45 e^{-02}\left( \pm 9.22 e^{-02}\right)$ \\
\hline VarY & $3.58 e^{-02}\left( \pm 4.98 e^{-02}\right)$ & $3.44 e^{-02}\left( \pm 4.73 e^{-02}\right)$ & $7.67 e^{-02}\left( \pm 1.01 e^{-01}\right)$ \\
\hline $\operatorname{Rad}$ & $5.55 e^{-02}\left( \pm 5.03 e^{-02}\right)$ & $4.96 \mathbf{e}^{-02}\left( \pm 5.06 e^{-02}\right)$ & $8.94 e^{-02}\left( \pm 6.91 e^{-02}\right)$ \\
\hline Ellips & $\mathbf{3 . 6 0 e ^ { - 0 1 }}\left( \pm 5.91 e^{-01}\right)$ & $3.71 e^{-01}\left( \pm 5.94 e^{-01}\right)$ & $1.21 e^{+00}\left( \pm 1.75 e^{+00}\right)$ \\
\hline
\end{tabular}

TABLE III: Mean error ( \pm standard deviation) of each approximations of the FBE family with respect to the KM, using the different metrics. The Butterworth filter and Optim-Boxcar were used with the parameters described in Subsection III.C.

\begin{tabular}{|c|c|c|c|c|}
\hline Metrics & LPFA & Butterworth Filter & Fixed-LPFA & Optim-Boxcar \\
\hline Pos & $6.61 e^{-02}\left( \pm 1.21 e^{-01}\right)$ & $8.43 e^{-02}\left( \pm 1.26 e^{-01}\right)$ & $6.50 \mathbf{e}^{-02}\left( \pm 1.21 e^{-01}\right)$ & $8.83 e^{-02}\left( \pm 1.25 e^{-01}\right)$ \\
\hline Spd & $1.32 e^{-03}\left( \pm 1.09 e^{-03}\right)$ & $2.21 e^{-03}\left( \pm 1.55 e^{-03}\right)$ & 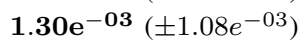 & $3.79 e^{-03}\left( \pm 2.41 e^{-03}\right)$ \\
\hline VarX & $1.91 e^{-02}\left( \pm 3.95 e^{-02}\right)$ & $2.13 e^{-02}\left( \pm 5.08 e^{-02}\right)$ & $1.69 \mathbf{e}^{-02}\left( \pm 3.77 e^{-02}\right)$ & $1.74 e^{-02}\left( \pm 4.36 e^{-02}\right)$ \\
\hline VarY & $4.77 e^{-02}\left( \pm 6.05 e^{-02}\right)$ & $3.95 e^{-02}\left( \pm 5.16 e^{-02}\right)$ & $4.33 e^{-02}\left( \pm 5.70 e^{-02}\right)$ & $3.32 \mathbf{e}^{-02}\left( \pm 4.71 e^{-02}\right)$ \\
\hline $\mathrm{Rad}$ & $4.60 e^{-02}\left( \pm 4.88 e^{-02}\right)$ & $5.55 e^{-02}\left( \pm 5.29 e^{-02}\right)$ & $4.43 e^{-02}\left( \pm 4.84 e^{-02}\right)$ & $5.75 e^{-02}\left( \pm 5.17 e^{-02}\right)$ \\
\hline Ellips & $4.71 e^{-01}\left( \pm 6.24 e^{-01}\right)$ & $4.54 e^{-01}\left( \pm 7.77 e^{-01}\right)$ & $4.06 e^{-01}\left( \pm 5.50 e^{-01}\right)$ & $3.34 e^{-01}\left( \pm 5.56 e^{-01}\right)$ \\
\hline
\end{tabular}

\section{B. Preprocessing}

Preprocessing, and in particular band-pass filters, are an essential component of the signals analysis. Different filter strategies have been proposed in past works, ranging from no filtering at all [19], to very restrictive filtering [28]. In our experiments, we observed that the choice of the cutoff frequency for $F_{h}$ had significant influence on the SI approximations performance (see Table I). As ZZDI appeared to outperform Global SI in most settings and to be more robust to the choice of the preprocessing, we chose to use ZZDI instead of Global SI in the following experiments.

We chose to apply a high-pass filter on $F_{h}$ with a 0.25 $\mathrm{Hz}$ cutoff frequency to remove accumulated integral error as in [27] and no filter on the CoM and the CoP trajectories in addition to the low-pass already applied to the dataset.

\section{Tuning Parameters}

In addition to the values suggested by their respective authors, we tested a range of parameters around them and selected the ones minimizing the mean norm of the difference between the positions of the true CoM and the one estimated.

- $\tau=0.3 \mathrm{~Hz}$ instead of $0.5 \mathrm{~Hz}$ for Fusion
- $0.45 \mathrm{~Hz}$ and $0.5 \mathrm{~Hz}$ for the cutoffs of the second order low-pass Butterworth filter for the medio-lateral and the antero-posterior axes.

- $\delta=0.6 \mathrm{~s}$ for the Boxcar model

\section{Metrics: Comparing CoM approximations}

In this experiment, we compare the CoM computed by all the approximations methods - using both the optimized and the recommended parameters - to the KM. In order to evaluate the pros and cons of the various approaches, we use a wide range of metrics defined below $\left(m_{t}, \hat{m}_{t}\right.$ and $x_{t}$ denote respectively the KM CoM, the approximate CoM and the CoP).

- The mean norm of the difference between the positions of $\hat{m}_{t}$ and $m_{t}$ (Pos) (unit: $\mathrm{cm}$ )

- The mean norm of the difference between the speeds of $\hat{m}_{t}$ and $m_{t}$ (Spd) (unit: $\mathrm{cm} . \mathrm{s}^{-1}$ )

- The mean of the absolute differences between the norms of deviation from the mean of $\hat{m}_{t}$ and $m_{t}$ (Rad) (unit: $\mathrm{cm})$

- The difference of the $95 \%$ confidence ellipse and both the medio-lateral and antero-posterior variances (three 
measures of the static balance quality [29]) between $\hat{m}_{t}$ and $m_{t}$ (resp. Ellips, VarX, VarY) (unit : $\mathrm{cm}^{2}$ )

The result of the different metrics are presented in Table II for the Force Integration (FI) family and Table III for the Frequency-Based Estimation (FBE) family. Overall, the FBE methods appeared to have the best performances, and FixedLPFA outperformed the other algorithms in most metrics, though by a slim margin. Figure 1 shows the trajectories of the CoM approximations generated by the two best methods (Fixed-LPFA and Optim-Fusion).

\section{Discussion}

Pros and cons of KM: While frequently considered the most accurate method, KM involves also the most time consuming acquisition protocol, and requires expensive sensors. Moreover the choice of the KM as the reference estimation for the CoM is arguable. Indeed, recent works have questioned the superior accuracy of the KM technique [17], namely due to errors in biodata measurements [13]. Previous papers have suggested that the combination of multiple approximations can produce a more reliable model [30].

Parameters tuning: In our experiments, we found that the best parameters for Boxcar, Butterworth and Fusion were different from the recommended values, and that optimizing the parameters lead to a significant increase in accuracy. However, the differences of values may be due to some hidden statistical properties of the respective datasets, therefore this observation should be reproduced in other datasets before drawing any conclusion.

Pros and cons of force integration methods: Compared to KM, FI methods do not require tracking markers and instead rely on the measurement of the ground reaction force in addition to the CoP trajectory. Nevertheless, FI methods performances appeared to be particularly dependent on the preprocessing choice. Among FI methods, Fusion appears to consistently outperform the others, in particular with tuned threshold parameter.

Pros and cons of frequency-based methods: FBE methods, except for LPFA, only require the recording of the CoP trajectory to compute the CoM approximation and still show good performance when compared to FI methods. This is a sizeable advantage, as it can be used together with low cost force platforms such as the Wii Balance Board. In contrast LPFA requires the recording of the height of the center of mass, which is not easily to acquire. Fixed-LPFA appears to avoid this issue while producting best accuracy in most metrics.

Signal preprocessing: It is important to note that the performances of the methods can be sensitive to preprocessing as shown in Table I. Therefore the experiments should be reproduced with other choices of preprocessing to lead to robust conclusions.

\section{CONCLUSION}

The alternative methods of the kinematic gold standard approximation of the center of mass, mostly based on force platforms, show interesting performances and require lower sensors cost and less time consuming protocols. In particular, Fixed-LPFA performs the best in most metrics in our results, while only requiring the recording of the center of pressure trajectory; therefore this method seems to be a tool of choice to estimate the CoM position and is a promising direction for further study.

\section{REFERENCES}

[1] "Important facts about falls," Centers for Disease Control and Prevention, Tech. Rep., 2017.

[2] D. A. Sterling, J. A. O'connor, and J. Bonadies, "Geriatric falls: injury severity is high and disproportionate to mechanism," Journal of Trauma and Acute Care Surgery, vol. 50, no. 1, pp. 116-119, 2001.

[3] M. E. Tinetti, "Preventing falls in elderly persons," New England journal of medicine, vol. 348, no. 1, pp. 42-49, 2003.

[4] M. Van Diest, C. J. Lamoth, J. Stegenga, G. J. Verkerke, and K. Postema, "Exergaming for balance training of elderly: state of the art and future developments," Journal of neuroengineering and rehabilitation, vol. 10, no. 1, p. 101, 2013.

[5] R. J. Peterka and M. S. Benolken, "Role of somatosensory and vestibular cues in attenuating visually induced human postural sway," Experimental brain research, vol. 105, no. 1, pp. 101-110, 1995.

[6] P. P. Perrin, C. Jeandel, C. A. Perrin, and M. C. Bene, "Influence of visual control, conduction, and central integration on static and dynamic balance in healthy older adults," Gerontology, vol. 43, no. 4, pp. 223-231, 1997.

[7] I. Melzer, N. Benjuya, and J. Kaplanski, "Postural stability in the elderly: a comparison between fallers and non-fallers," Age and ageing, vol. 33, no. 6, pp. 602-607, 2004.

[8] D. Lafond, M. Duarte, and F. Prince, "Comparison of three methods to estimate the center of mass during balance assessment," Journal of biomechanics, vol. 37, no. 9, pp. 1421-1426, 2004.

[9] M. Piirtola and P. Era, "Force platform measurements as predictors of falls among older people-a review," Gerontology, vol. 52, no. 1, pp. $1-16,2006$

[10] O. Caron, T. Gélat, P. Rougier, and J.-P. Blanchi, "A comparative analysis of the center of gravity and center of pressure trajectory path lengths in standing posture: an estimation of active stiffness," Journal of Applied Biomechanics, vol. 16, no. 3, pp. 234-247, 2000.

[11] L. Baratto, P. G. Morasso, C. Re, and G. Spada, "A new look at posturographic analysis in the clinical context: sway-density versus other parameterization techniques," Motor control, vol. 6, no. 3, pp. 246-270, 2002.

[12] R. A. Clark, A. L. Bryant, Y. Pua, P. McCrory, K. Bennell, and M. Hunt, "Validity and reliability of the nintendo wii balance board for assessment of standing balance," Gait \& posture, vol. 31, no. 3, pp. 307-310, 2010.

[13] D. Lenzi, A. Cappello, and L. Chiari, "Influence of body segment parameters and modeling assumptions on the estimate of center of mass trajectory," Journal of biomechanics, vol. 36, no. 9, pp. 13351341, 2003.

[14] M. Saini, D. Kerrigan, M. Thirunarayan, and M. Duff-Raffaele, "The vertical displacement of the center of mass during walking: a comparison of four measurement methods," Journal of biomechanical engineering, vol. 120, no. 1, pp. 133-139, 1998.

[15] J. Eng and D. Winter, "Estimations of the horizontal displacement of the total body centre of mass: considerations during standing activities," Gait \& Posture, vol. 1, no. 3, pp. 141-144, 1993.

[16] B. J. Benda, P. O. Riley, and D. E. Krebs, "Biomechanical relationship between center of gravity and center of pressure during standing," IEEE Transactions on Rehabilitation Engineering, vol. 2, no. 1, pp. 3-10, 1994.

[17] M. A. Thirunarayan, D. C. Kerrigan, M. Rabuffetti, U. Della Croce, and M. Saini, "Comparison of three methods for estimating vertical displacement of center of mass during level walking in patients," Gait \& Posture, vol. 4, no. 4, pp. 306-314, 1996.

[18] H. M. Schepers, E. H. Van Asseldonk, J. H. Buurke, and P. H. Veltink, "Ambulatory estimation of center of mass displacement during walking," IEEE Transactions on Biomedical Engineering, vol. 56, no. 4, pp. 1189-1195, 2009.

[19] T. Shimba, "An estimation of center of gravity from force platform data," Journal of biomechanics, vol. 17, no. 1, pp. 59-60, 1984. 
[20] V. M. Zatsiorsky and D. L. King, "An algorithm for determining gravity line location from posturographic recordings," Journal of biomechanics, vol. 31, no. 2, pp. 161-164, 1997.

[21] O. Caron, B. Faure, and Y. Brenière, "Estimating the centre of gravity of the body on the basis of the centre of pressure in standing posture," Journal of biomechanics, vol. 30, no. 11-12, pp. 1169-1171, 1997.

[22] D. A. Dos Santos, C. A. Fukuchi, R. K. Fukuchi, and M. Duarte, "A data set with kinematic and ground reaction forces of human balance," PeerJ, vol. 5, p. e3626, 2017.

[23] P. De Leva, "Adjustments to zatsiorsky-seluyanov's segment inertia parameters," Journal of biomechanics, vol. 29, no. 9, pp. 1223-1230, 1996.

[24] R. Chan, "A method for estimating center of mass from forceplate data during quiet standing," in [Engineering in Medicine and Biology, 1999. 21st Annual Conference and the 1999 Annual Fall Meetring of the Biomedical Engineering Society] BMES/EMBS Conference, 1999. Proceedings of the First Joint, vol. 1. IEEE, 1999, pp. 516-vol.

[25] Y. Brenière, "Why we walk the way we do," Journal of motor behavior, vol. 28, no. 4, pp. 291-298, 1996.

[26] P. Rougier, C. Burdet, I. Farenc, and L. Berger, "Backward and forward leaning postures modelled by an fbm framework," Neuroscience Research, vol. 41, no. 1, pp. 41-50, 2001.

[27] M. Brodie, A. Walmsley, and W. Page, "Fusion integration: Com trajectory from a force platform," Journal of Applied Biomechanics, vol. 23, no. 4, pp. 309-314, 2007.

[28] O. Levin and J. Mizrahi, "An iterative model for estimation of the trajectory of center of gravity from bilateral reactive force measurements in standing sway," Gait \& posture, vol. 4, no. 2, pp. 89-99, 1996.

[29] R. J. Doyle, E. T. Hsiao-Wecksler, B. G. Ragan, and K. S. Rosengren, "Generalizability of center of pressure measures of quiet standing," Gait \& posture, vol. 25, no. 2, pp. 166-171, 2007.

[30] H.-M. Maus, A. Seyfarth, and S. Grimmer, "Combining forces and kinematics for calculating consistent centre of mass trajectories," Journal of Experimental Biology, vol. 214, no. 21, pp. 3511-3517, 2011 